\title{
Le Projet de Corpus des Mosaïques d'Albanie Arnavutluk Mozaikleri Korpus Projesi
}

Marie-Patricia RAYNAUD - Agron ISLAMI*

(Received 04 March 2015, accepted after revision 12 November 2015)

\begin{abstract}
After the issue of the first two volumes of the Corpus of the mosaics of Turkey, an Albanian Corpus was initiated in 2013, based on similar norms. Six to eight volumes should be necessary to publish the whole pavements of the country. The relatively limited surface of the area already allows considerations about local or itinerants workshops, about sponsors and economics assets, circulation of repertory and technical habits. A team is been trained to perform study, layout and restoration altogether on the field.
\end{abstract}

Keywords: Mosaïque, Turquie, Albanie, Antiquité, Byzance.

\section{Öz}

Illk iki ciltten oluşan "Türkiye Mozaikleri” konulu korpusun tamamlanmasından sonra, 2013 yllında başlatılan Arnavutluk Korpusu da benzer normları baz almıștır. Yayınlanacak altı ile sekiz cildin içeriği, ülkenin tüm döşemelerini ele alacaktır. Bölgenin nispeten sınırlı alanını kapsayan çalışma, yerli ve gezici atölyeler, sponsorlar ve ekonomik varlıkları, repertuvarın dolaşımı ve teknik alışkanlıklar hakkındaki düşüncelere olanak tanır. Bir ekip, alanda çalışmayı, restorasyonu ve düzeni gerçekleştirmek için hep birlikte eğitilmiştir.

Anahtar Kelimeler: Mozaik, Türkiye, Arnavutluk, Antik, Bizans.

La création du Corpus des mosaïques de Turquie, les premières réunions régulières de ses membres et la mise en place de ses normes (Morlier - Raynaud 2007) ont été, pour la plupart des fondateurs, une riche et belle aventure. Il y régnait une ambiance de confiance, d'amitié et un goût du partage, et ces moments étaient festifs et stimulants. Beaucoup d'entre nous sont restés amis, et en contact depuis lors. Décédé récemment, Barış Salman a dès 2006 participé aux symposiums annuels, il était un membre important du Corpus et nous ne l'oublierons pas.

Si l'aventure a commencé dès 2004 à Istanbul, l'Association AIEMA Türkiye s'est réellement mise en place en 2005 à Izmir et Altınoluk (Antandros, Balıkesir). Puis en 2006 à Bursa, les normes de publication et un modèle de fiche descriptive y ont été proposés et adoptés, et le meeting de l'AIEMA Türkiye a pour la première fois pris l'allure d'un symposium dont les communications furent publiées. Les meetings suivants, Gaziantep (2007), Karamanmaraş (2010) et Bursa (2014), ont connu un succès croissant. Les publications des actes se sont développées, pour finir par être prises en charge par le Journal of Mosaic Research (JMR, premier volume en 2008), revue internationale de qualité, créée par le Corpus de Turquie et son président Mustafa Şahin ${ }^{1}$. Enfin le succès du colloque international sur la mosaïque (Colloque AIEMA) tenu à Bursa en 2009, et magnifiquement publié en 2011, a achevé de montrer aux spécialistes de la mosaïque la vitalité de la branche turque. Après la création, grâce à Hélène Morlier, d'une maquette «sur mesure » pour la série du Corpus en 2008-09, deux volumes sur Xanthos (Lycie) ont déjà vu le jour

\footnotetext{
* Marie-Patricia RAYNAUD, CNRS (Centre national de la Recherche scientifique), Orient et Méditerranée (UMR 8167), Le monde de Byzance, 52 rue du Cardinal Lemoine, 75005 Paris. E-mail: marie-pat.raynaud@ college-de-france.fr ; Agron ISLAMİ, Instituti i Monumenteve të Kulturës (IMK), Rr. Aleksander Moisiu, nr. 76, Kinostudio, Tirana, Albanie. E-mail: gonislami@yahoo.it

1 Nous apprécions tous la qualité de la mise en page et une excellente qualité d'illustration en couleur de cette revue au comité scientifique international. Le colloque de Kahramanmaraş (2010) a fait l'objet du volume 5, en 2012. Le volume 8 sera le volume d'hommages à Barış Salman.
} 
(Raynaud 2009; Manière-Lévêque - Parrish - Raynaud 2012), d'autres suivront, nous l'espèrons bien vivement.

Il aurait été dommage de ne pas mettre à profit l'expérience acquise en Turquie pour la création d'autres corpus. Nous avons donc souhaité en 2013 lancer cette entreprise dans un pays, l'Albanie, dont les pavements sont encore très mal connus.

\section{Création du Corpus d'Albanie}

Il a été donc décidé dès 2012 de créer un Corpus des mosaïques d'Albanie, sous la direction de A. Islami et de M.-P. Raynaud. Une convention entre nos deux pays a été signée en 2013 par le CNRS, l'IMK et l'université Paris IV.

Travaillant ensemble depuis 1999 sur divers sites, il nous apparaissait tout naturel et souhaitable de continuer de mettre en commun nos diverses compétences, qui avaient permis des avancées intéressantes sur le terrain ${ }^{2}$, pour produire un inventaire le plus complet possible des nombreuses mosaïques découvertes. L'accès à tous les pavements d'Albanie est désormais facilité, grâce à la caution de l'IMK et sous la responsabilité de son restaurateur en chef. Sur le terrain, l'étude et la documentation (photographies, plans, relevés) sont menés de front avec un contrôle de l'état de conservation, accompagné de campagnes de restauration et d'une surveillance sévère de la pose d'une protection adaptée à chaque pavement. Ce travail in situ est complété par un travail en archives afin de réunir toute la documentation nécessaire au chapitre historiographique sur chaque monument.

Une équipe internationale s'est peu à peu mise en place et continue à s'étoffer, avec restaurateurs (A. Islami, A. Maréchaux, Kl. Zgurro ${ }^{3}$ ), chercheurs spécialisés (M.-P. Raynaud, Y. Marano, E. Neri, M. Tutkovski ${ }^{4}$ ), photographe (D. Dubois ${ }^{5}$ ) et étudiants. Chacun, s'il le souhaite, est formé aux différentes techniques de relevés graphiques, aux photographies spécifiques (orthophotographies avec un monopode, photographies d'ensemble au drone, photographies macro et au microscope digital pour l'étude des matériaux) ainsi qu'éventuellement aux techniques de base de la conservation in situ des pavements. L'initiation se poursuit avec les descriptions des pavements grâce au vocabulaire mis au point par le Centre H. Stern (CNRS Paris) ${ }^{6}$, proposé en cinq langues dans deux volumes traitant du décor géométrique des mosaïques (Décor I 1985; Décor II 2002). Enfin ceux qui le désirent peuvent éventuellement se former à la finalisation des illustrations pour la publication.

2 Nous avons plusieurs fois pu constater combien le travail simultané du restaurateur et du chercheur pouvait apporter à la compréhension de la technique des mosaïstes et aboutir à des découvertes aidant à établir la chronologie des pavements ; ainsi fut découverte à Byllis l'existence d'une maison romaine antérieure à la basilique B : (Islami - Raynaud 2014).

3 Restaurateurs de mosaïques tous les deux, Astrid Maréchaux exerce en France, Klejdi Zguro à Tirana.

4 Yuri Marano, post-doctorant au Labex Resmed en 2014, étudie les tombes privilégiées lorsque ces dernières sont liées aux mosaïques étudiées ; Elisabetta Neri, Post-doc au Labex Resmed en 2014, à l'IdexSuper Convergence (programme Orluce) en 2015, est spécialiste des mosaïques pariétales et de l'analyse archéométrique du verre ; Miško Tutkovski, jeune conservateur à Stobi, a fait un ouvrage sur les mosaïques d'Ohrid.

5 Didier Dubois, topographe et photographe en archéologie, réalise les prises de vues du Corpus et participe aux relevés.

6 Ces ouvrages collectifs illustrés, publiés à Paris, donnent les règles de description multilingues pour tous les décors de la mosaïque géométrique, avec en regard le dessin du décor correspondant, permettant l'usage d'un vocabulaire univoque et compréhensible par tous les spécialistes de la discipline. 
À l'échelle d'un pays comme l'Albanie, le projet de publier les mosaïques découvertes à ce jour pourrait aboutir avec la publication de six à huit volumes, tache réalisable par une même équipe en un temps relativement raisonnable. C'est pourquoi la formation de ses membres nous a d'emblée semblé essentielle, afin que les méthodes employées restent uniformes et pour garantir un même niveau de qualité.

\section{Institutions participantes}

Les Institutions qui participent actuellement au soutien scientifique et au polyfinancement du projet sont les suivantes:

- L'IMK (Institut des monuments culturels, Tirana), par la participation de salariés, l'accès aux archives et l'organisation logistique des missions.

- Le Labex RESMED (Religions et sociétés dans le monde méditerranéen) pour sa section A, Religions, lieux et conflits : 2. Paysages et édifices), apporte largement son concours aux missions, et sera sollicité lors la publication des volumes du Corpus.

- L'équipe du CNRS «Monde byzantin », UMR 8167 (Orient et Méditerranée), participe aux missions et soutient le travail de préparation des publications. Elle accueillera les réunions de publication qui auront lieu à Paris.

- L'Université Sorbonne-Paris IV collabore au projet par le financement de missions.

- Des associations (Butrint Foundation, Butrint Heritage Center), des entreprises privées (de tourisme, du bâtiment, banques, etc.) et des mécènes (soutien financier à la mise au point d'un drone spécifique pour la photogrammétrie de mosaïque) sont ou seront sollicités en fonction des sites concernés ou des travaux engagés (fourniture de matériaux, crédits d'analyse et de publication). La Butrint Foundation a généreusement subventionné les campagnes de 2013 à 2015 sur ce site.

- L'EfR (École française de Rome) et l'EfA (École française d'Athènes), contactées, ont été favorables au projet et se sont engagées à soutenir les travaux du Corpus sur les sites albanais où ces institutions sont implantées (Byllis et Apollonia).

- L'Ambassade de France à Tirana, informée du projet dès 2013, pourra collaborer à des opérations de valorisation ponctuelles sur les sites ou à des expositions.

- L'IAT (Institut archéologique de Tirana) participe à travers la consultation des archéologues de chaque site. Les archives de l'IAT devront être compulsées systématiquement.

\section{Les mosaïques d'Albanie}

Les mosaïques d'Albanie sont très inégalement connues. Elles constituent un riche patrimoine, dense et de qualité, souvent peu publié, parfois encore ignoré et dans la majeure partie des cas invisible (Fig. 1). La conservation et la mise en valeur de ce Patrimoine archéologique exceptionnel font partie des priorités de la politique culturelle albanaise. La publication scientifique constitue une étape décisive pour mettre ces mosaïques restaurées à la disposition d'un public de plus en plus large. Très fréquemment, les mosaïques constituent les vestiges les mieux conservés des monuments ruinés. Leur étude et leur présentation dans 


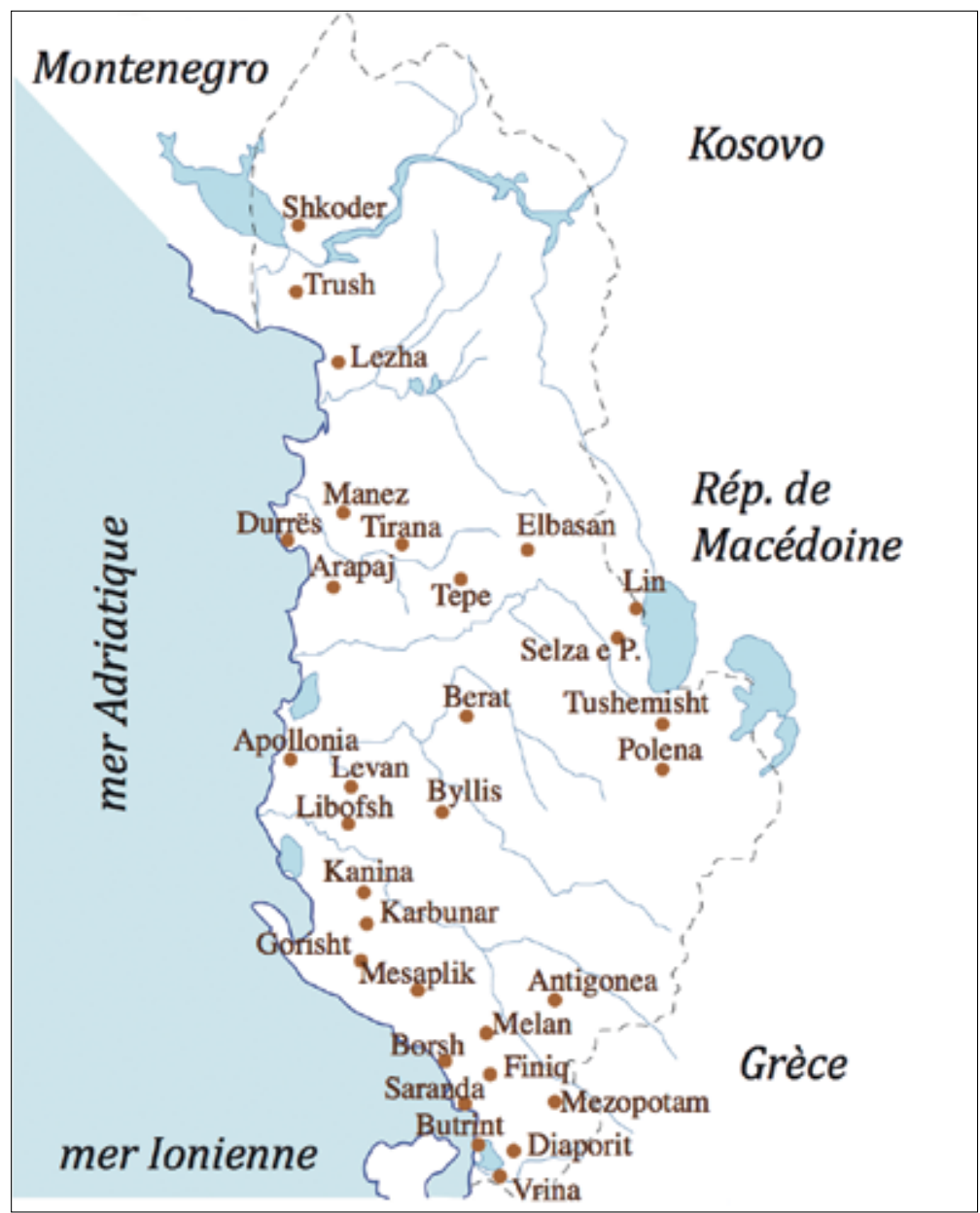

Figure 1

Carte de répartition des mosaïques d'Albanie (M.-P. Raynaud).

leur contexte architectural permettent de mieux appréhender l'histoire, la chronologie et l'insertion des édifices dans un environnement régional.

Les mosaïques d'Albanie ont souvent été mises au jour dans ou à proximité des villes, parfois lors de fouilles urbaines de sauvetage qui ne donnent qu'une idée partielle des édifices auxquels elles appartenaient. D'autres appartiennent à des sites antiques en cours de fouilles, et il est probable que de nouvelles découvertes auront lieu dans les années à venir.

S'il existe quelques mosaïques hellénistiques ${ }^{7}$, les pavements d'époque romaine sont plus répandus (thermes, maisons), particulièrement sur la zone littorale ${ }^{8}$. L'Antiquité tardive est cependant la période la plus riche. La géographie de l'Église de l'Épire et de la Macédoine, de la fin du ve jusqu'au troisième quart $\mathrm{du} \mathrm{VI}^{\mathrm{e}}$ siècle, reflète une fourmillante période de prospérité, arrivée à son sommet à l'époque de Justinien. En sont témoins les multiples monuments chrétiens qui ont couvert ces territoires. Ces bâtiments, fréquemment construits de novo, sont édifiés soit dans de grands sites urbains et ecclésiastiques ${ }^{9}$, soit dans des

7 Mosaïques hellénistiques découvertes à Butrint (Buthrotom), Saranda (Onchesmos), Durrës (Dyrrhachium) et Apollonia.

8 Sur les mêmes sites que les mosaïques hellénistiques.

9 Butrint, Saranda, Apollonia, Durrës, Lezha (Lissos), Shkodra, Elbasan (Scampis), Byllis. Citons pour les régions limitrophes hors d'Albanie (ne faisant donc pas partie du Corpus), les sites d'Ohrid (Lychnidos), Héraklea Lyncestis, Stobi et Nicopolis. 

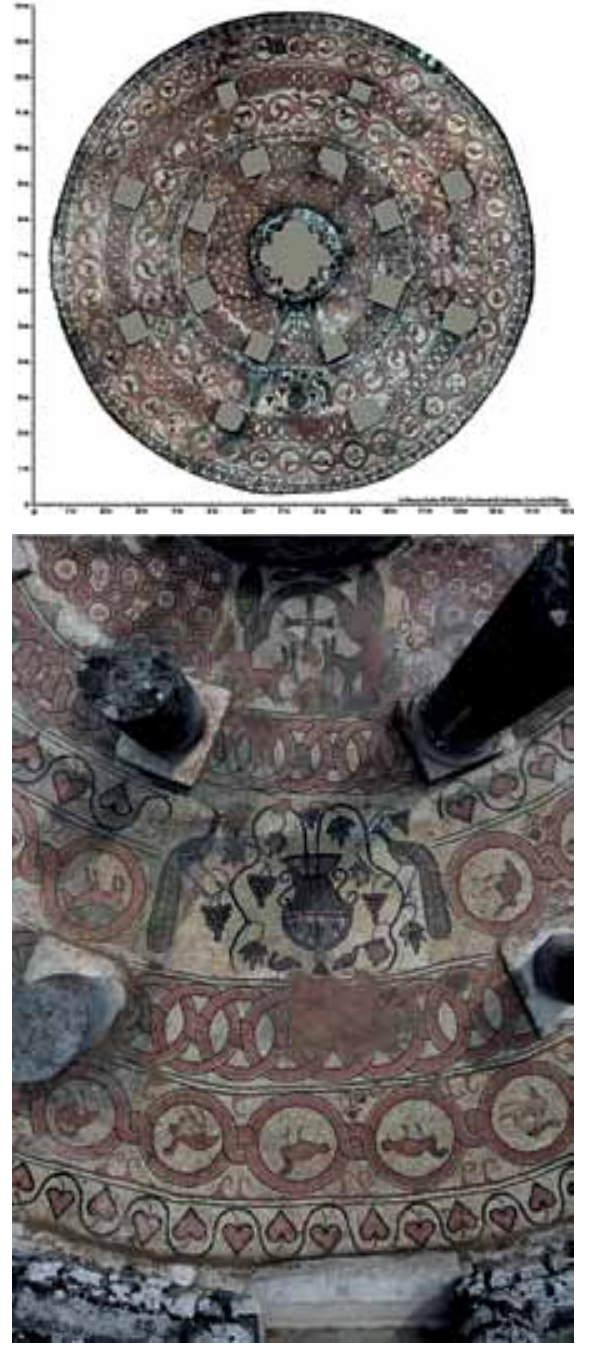

Figure 2

Butrint, baptistère, vue d'ensemble (M. Zanfini) et panneaux d'entrée (D. Dubois). petites villes rurales ${ }^{10}$. Les nombreux pavements de ces églises illustrent l'importance des voies qui sillonnaient le pays, en particulier l'axe de la via Egnatia qui traversait l'Albanie actuelle d'Ouest en Est, voie directe entre Rome et Constantinople, favorisant la circulation des idées, des répertoires et des équipes d'artisans.

Mentionnons à Saranda le cas d'une synagogue transformée en église au $\mathrm{VI}^{\mathrm{e}}$ siècle, où l'on observe l'exemple rare d'une conversion apparemment sans heurts. Peu d'exemples d'habitat de l'Antiquité tardive ont été mis au jour à part le complexe du Triconque de Butrint, et il semble, dans l'état actuel des connaissances, que l'art de la mosaïque étaient généralement réservé à la décoration des lieux de culte.

Le contenu de chaque volume du Corpus s'adaptera à la densité des pavements considérés. La plupart seront consacrés à un site, dans la mesure où son importance le permet et les pavements seront analysés chronologiquement par édifices.

Un premier volume concernera les mosaïques intra muros de Butrint, toutes époques confondues (Hodges - Bowden - Lako 2004; Bowden - Hodges 2011; Hansen - Hodges - Leppard 2013) ${ }^{11}$. La mosaïque hellénistique du temple d'Asklépios, récemment retrouvée par l'équipe du Corpus (Marika - Nesturi 2001: 14), offrant une représentation de serpent utilisant la technique des lames de plomb pour en souligner le contour, fera l'objet d'une étude particulière. Les mosaïques romaines, provenant principalement de thermes et monuments publics, attestent pour la plupart le travail d'un même atelier prolifique dont on commence à cerner le profil. Enfin l'Antiquité tardive est bien représentée par les mosaïques du complexe au Triconque, de la grande basilique, de la basilique de l'Acropole et naturellement du grand Baptistère circulaire (Fig. 2) (Mitchell 2008).

Le ou les volume(s) sur Durrës (Hoti - Shehi - Santoro 2012) ${ }^{12}$ et Apollonia (Dimo - Lenhardt - Quantin 2007; Skenderaj 2014) ${ }^{13}$ seront plus riches pour les époques hellénistiques et romaines où ces villes ont prospéré, avec en particulier la reprise de l'étude du pavement en galets de la «Belle de Durrës ${ }^{14}{ }^{\text {» }}$, des mosaïques romaines de la maison d'Athéna (Fig. 3) et de la maison $\mathrm{G}$ à Apollonia, qui n'ont fait l'objet ni de relevés détaillés, ni d'étude systématique. Plusieurs pavements de ces sites sont inédits ou parfois simplement cités sans illustration par les chroniques de fouilles de sauvetage publiées dans les revues albanaises ; ils seront systématiquement analysés et documentés. Les restaurateurs pourront à cette occasion contrôler leur état de conservation, intervenir au besoin et renouveler la protection de ces sols anciennement découverts.

Enfin, le ou les volumes concernant Byllis seront principalement dévolus aux mosaïques des cinq églises protobyzantines (Chevalier et al.2003) ${ }^{15}$ des ve-début

10 Par exemple Arapaj, Antigonea, Melan, Mesaplik, Tepe Elbasan, Lin.

11 Pour certains édifices déjà bien publiés, l'étude architecturale sera allégée.

12 L'expansion urbaine de Durrës durant les cinquante dernières années a été à l'origine de nombreuses interventions ponctuelles de sauvetage sur ce secteur littoral convoité (voir les nombreuses publications du « Projetto Durrës », projet italo-albanais qui y travaille depuis une dizaine d'années). Un bilan systématique, principalement documenté par les archives, donnerait un meilleur aperçu de ces mosaïques fragmentaires, éparses et souvent perdues.

13 L'Atlas signale brièvement les mosaïques. Quelques guides mentionnent les mêmes photographies d'archives ; l'article de A. Skenderaj offre une bonne documentation d'archives.

14 Mosaïque hellénistique conservée au musée historique national de Tirana (cf. Guimier-Sorbet 1993).

15 En 1999, une équipe franco-albanaise s'est réunie autour de Skënder Muçaj et Jean-Pierre Sodini puis Pascale Chevalier, sous l'égide de l'École française d'Athènes, afin de compléter la documentation et de publier les fouilles menées à Byllis (Albanie) dans les années 1980 par Sk. Muçaj. Cette coopération 

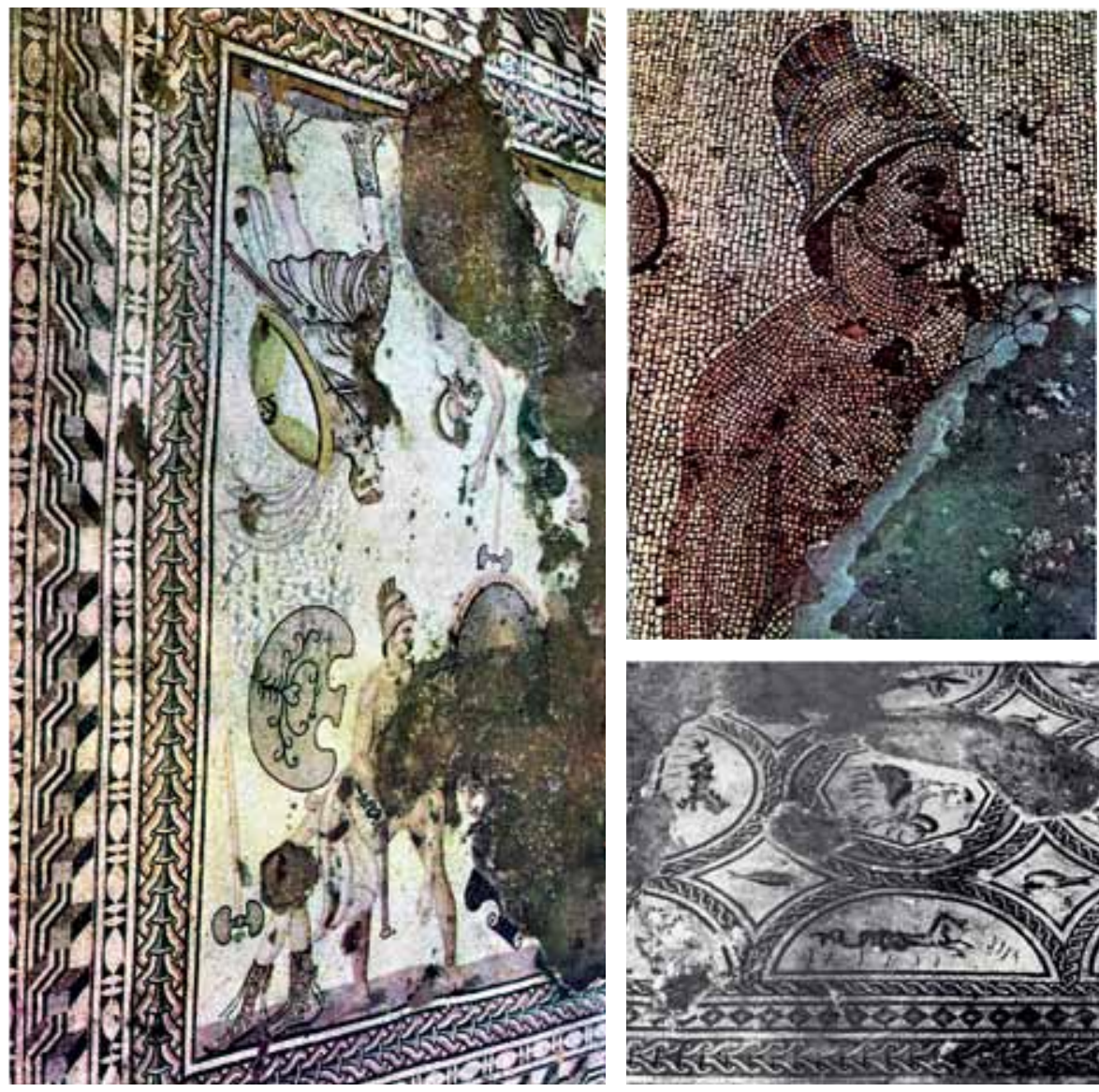

Figure 3

Apollonia, maison d'Athéna, mosaïque d'Achille et pavement des Néréides

(Archives IMK).

$\mathrm{VI}^{\mathrm{e}}$ s. plus de $1000 \mathrm{~m}^{2}$, en bon état de conservation (Fig. 4), dont le répertoire figuré varié témoigne d'une uniformité et d'une maîtrise peu fréquentes (MuçajRaynaud 2005; Raynaud 2014). On y adjoindra les quelques mosaïques romaines ou funéraires découvertes sur le site. L'étude de terrain est presque terminée, une campagne de photographies au drone est prévue. D'autres mosaïques du centre de l'Albanie pourront être rattachées à ces volumes, comme celles de Berat (Bushi - Xhaferaj - Behari 2014), d'Antigonea(Budina - Korkuti 1971; Mitchell 2006) et de Mesaplik (Komata 1984) dans la région de Vlora.

D'autres volumes seront consacrés aux mosaïques d'une région entière : le volume de la région de Saranda, par exemple, comprendra principalement l'analyse de la synagogue intramuros transformée en église (Fig. 5) (Nallbani 2003; Foerster et al. 2004; Nallbani et al. 2011 ${ }^{16}$. La chronologie relative des

rassemblait des spécialistes de divers domaines, et nous avons été sollicités pour les pavements du site, M.-P. Raynaud pour l'étude (chapitres sur la mosaïque) et A. Islami pour la restauration des mosaïques. Plusieurs campagnes sur le site ont permis d'enregistrer une documentation complète. Le premier volume de la publication des fouilles, déposé en 2008 à l'EfA, est en cours d'impression, il concerne les fortifications byzantines et les églises A, C et D. Le second volume concernera la cathédrale (ou basilique B) et le quartier épiscopal et sera suivi par l'étude de la basilique E et du matériel récolté pendant les fouilles.

16 Après des fouilles des années 1980, une reprise des travaux eut lieu en 2003 à la suite de la découverte dans les archives par Etleva Nallbani d'une représentation en mosaïque de menorah. La remise en question de l'identité religieuse du complexe (jusqu'alors interprété comme église) a réuni autour d'elle et de Kosta Lako, l'archéologue qui avait fouillé l'ensemble dans les années 1980, deux archéologues et architectes israéliens Gideon Foerster et Ehud Netzer, auxquels se sont joints, entre autres, M.-P. Raynaud pour l'étude des sols et A. Islami pour les restaurations. Plusieurs campagnes de fouilles et d'étude ont eu lieu entre 2003 et 2009, la publication définitive des travaux est en cours. Des analyses archéométriques des tesselles sont actuellement réalisées. 

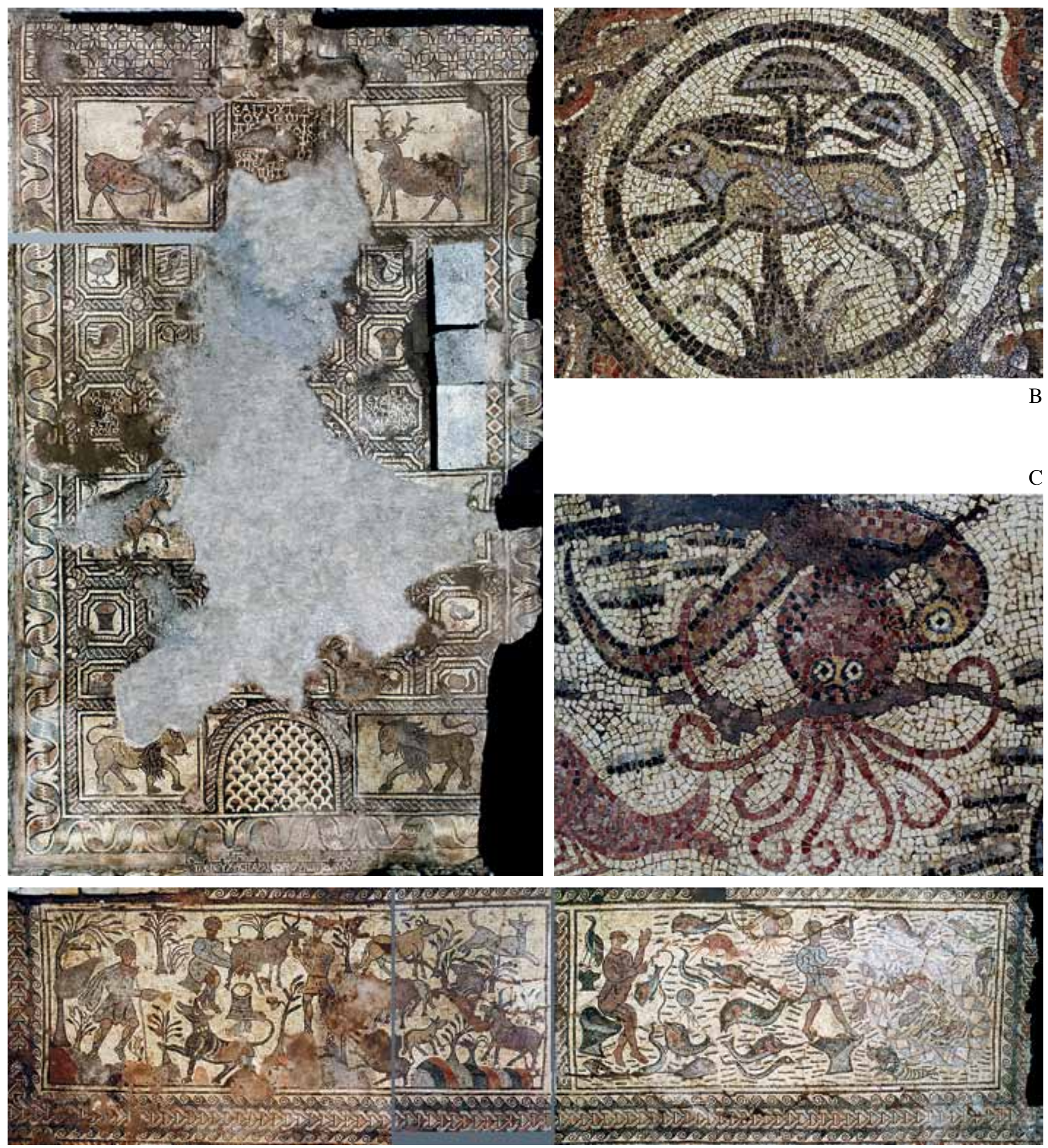

Figure 4

Byllis, A-nef de la basilique D ; basilique B : B et C-détails ;

D-exonarthex (M.-P. Raynaud, D. Dubois). pavements successifs a été étudiée et permet une meilleure compréhension de ce complexe urbain (Raynaud 2012). On adjoindra à ce volume l'analyse des pavements romains trouvés dans ce port d'importance, ainsi que les mosaïques d'établissements romains et d'églises protobyzantines des environs de Butrint, dans la plaine de Vrina (Greenslade - Çondi 2010; Greenslade 2013) ${ }^{17}$ et à

17 Une grande basilique protobyzantine y a été découverte, dont la nef est entièrement mosaïquée. Des mosaïques d'époque romaine ont également été mises au jour dans un quartier d'habitation antérieur. 

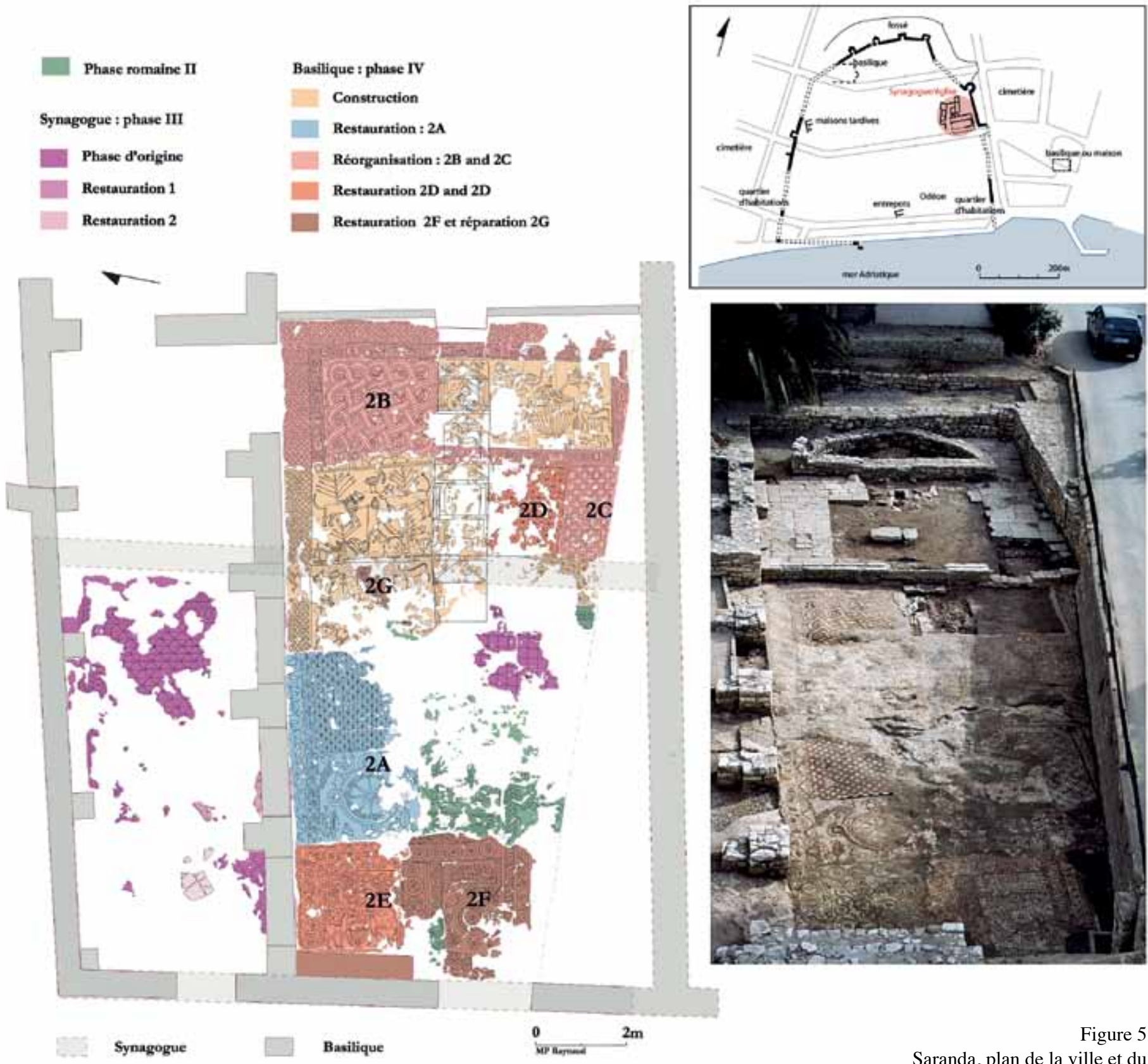

Diaporit (Bowden 2007; Përzhita - Bowden 2011; Bowden - Përzhita 2014), ainsi que les vestiges trouvés à Phoinike (De Maria - Zaccaria 2005; Podini Meta - Silani 2007; De Maria - Podini 2009).

Couvrant un secteur plus large, un des volumes rassemblera les mosaïques pratiquement inédites du Nord et de l'Est du pays, datant principalement de l'époque romaine, comme celles de Lezha (Lissos, bains romains) et de Shkodra (Scuteri, mosaïques présentées en petites plaques dans le musée de la forteresse de Rozafa), celles de Tirana (villa romaine tardive, avec un triclinium à abside mosaïqué), le fragment de Polena (au musée de Korça) et des morceaux trouvés au cours de fouilles de sauvetage (archives).

Certains volumes seront plus thématiques. L'un d'entre eux prendra en compte l'étude des églises protobyzantines placées le long de la via Egnatia, comprenant les basiliques d'Arapaj près de Durrës (Fig. 6) (Hidri - Hidri 2011; 
Figure 6

Arapaj, panneaux de mosaïque placés au-dessus de la tombe (d'ap. Hidri - Hidri 2011: abb. 31-32).
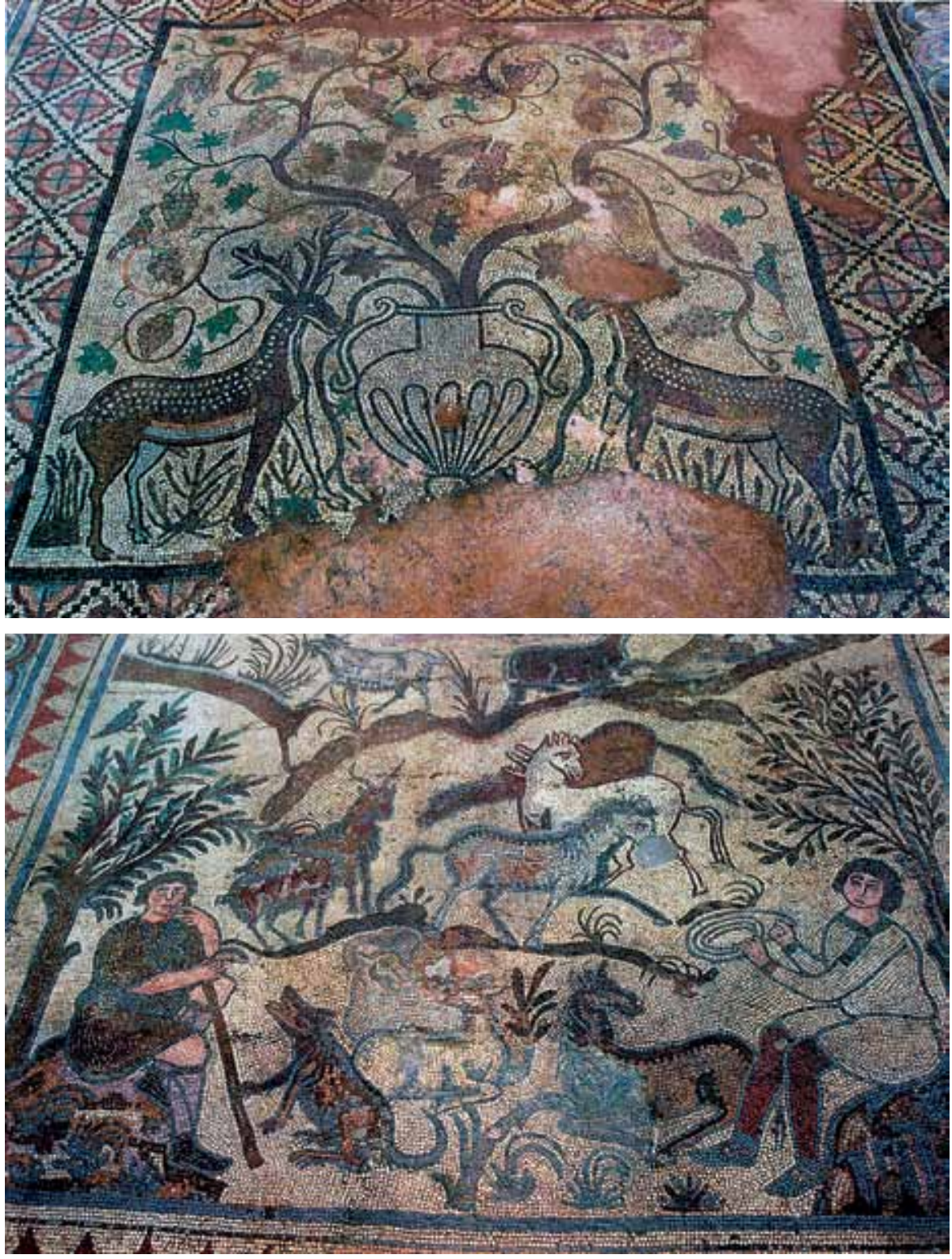

Omari 2006; Omari 2012), Elbasan ${ }^{18}$ (Fig. 7) (Cerova - Hobdari - Islami 2011; Hobdari - Cerova 2013), Tepe Elbasan (Meksi - Ceka 1971; Kosta - Çikopano 1990) et Lin (Anamali 1974). La plupart sont des églises à vocation martyriale, comme celle de Lin, située au bord du lac d'Ohrid (Fig. 8). Les multiples similitudes de cette dernière avec les nombreuses églises et mosaïques des alentours d'Ohrid (République de Macédoine, Bitrakova-Grozdanova et al. 1975; Tutkovski 2014) nous incitent à entreprendre leur étude avec nos collègues de Macédoine, ce qui facilitera les comparaisons.

Un fascicule regroupera les pavements en opus sectile et médiévaux d'Albanie, en particulier les intéressantes rosaces de pierre et briques observées dans les

18 Les mosaïques de cette église nouvellement fouillée, à la fois murales et de pavement, sont remarquables par leur qualité d'exécution, leur iconographie et la quantité de tesselles en verre employées. Les disparités stylistiques et techniques entre les sols du narthex étudié par le corpus en 2013 et de la nef (fouille inachevée) attestent le travail de divers ateliers en plusieurs phases. L'analyse comparée des matériaux des mosaïques de sols et des fragments pariétaux d'Elbasan devrait préciser si ces mosaïques sont l'œuvre d'une même équipe. 


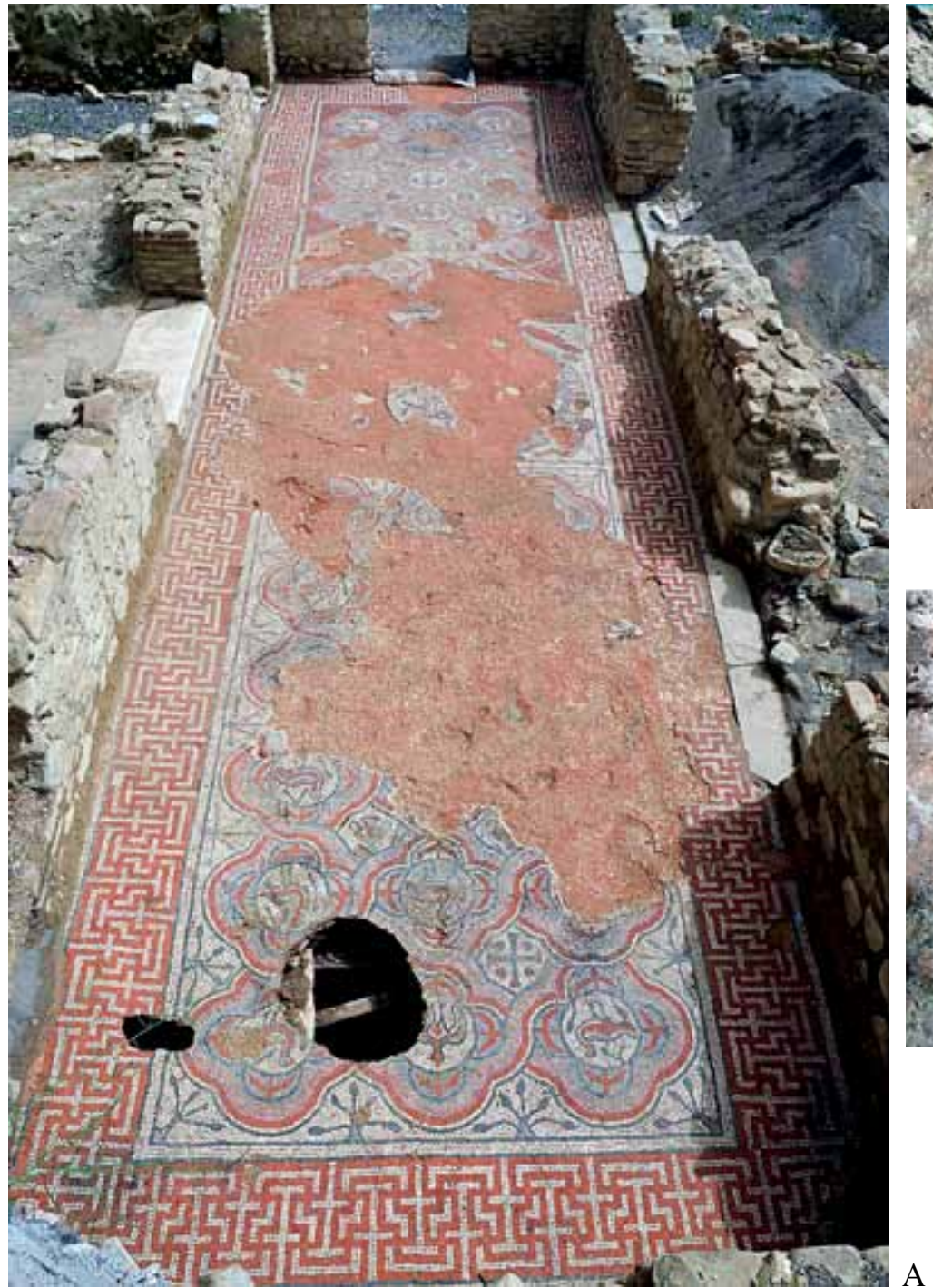

églises de Berat, et de pierres du Sud de l'Albanie, à Pëshkepi (Muçaj - Hobdari - Vitaliotis 2005) et dans l'église de Shën Jan à Delvina (Muçaj et al. 2004), auxquelles seront ajoutés les panneaux de mosaïques médiévales de l'église de Mesopotam, conservés à Korça.

L'Albanie n'a révélé que quelques mosaïques pariétales ; elles seront étudiées dans les volumes correspondant au site dont elles proviennent : il s'agit des mosaïques des niches du « Gymnasium » de Butrint, de la chapelle de l'amphithéâtre de Durrës (Fig. 9) et des centaines de fragments récoltés lors des fouilles de l'église d'Elbasan en 2012.

Pour les années à venir, le suivi, par l'équipe du Corpus, des prochaines découvertes dès leur fouille garantira un meilleur enregistrement des données spécifiques aux mosaïques, permettra l'analyse immédiate des matériaux et mortiers, actions plus délicates à mener lorsque les restaurations ont déjà eu lieu.
Figure 7

Elbasan, basilique extra muros, A-narthex, avec une tombe sousjacente au premier plan (D. Dubois); $\mathrm{B}$ et C-mosaïque de la nef pendant les fouilles (E. Hobdari, A. Islami). 
Figure 8

Lin, plan de l'église et des sols (en vert les mosaïques, en orange les carreaux de terre cuite ; M.-P. Raynaud); -mosaïque de la chapelle martyriale et de l'annexe Sud (D. Dubois).
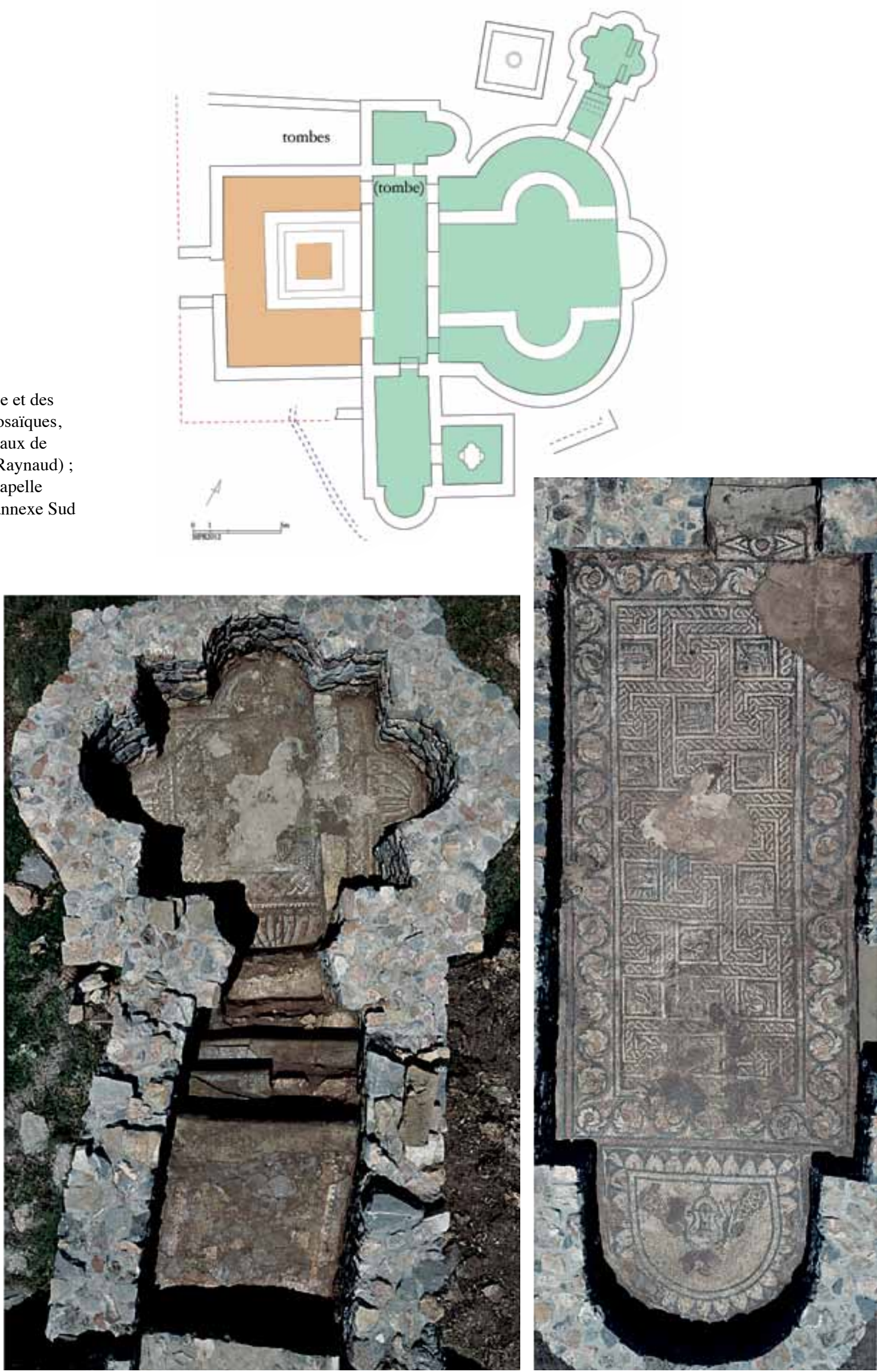


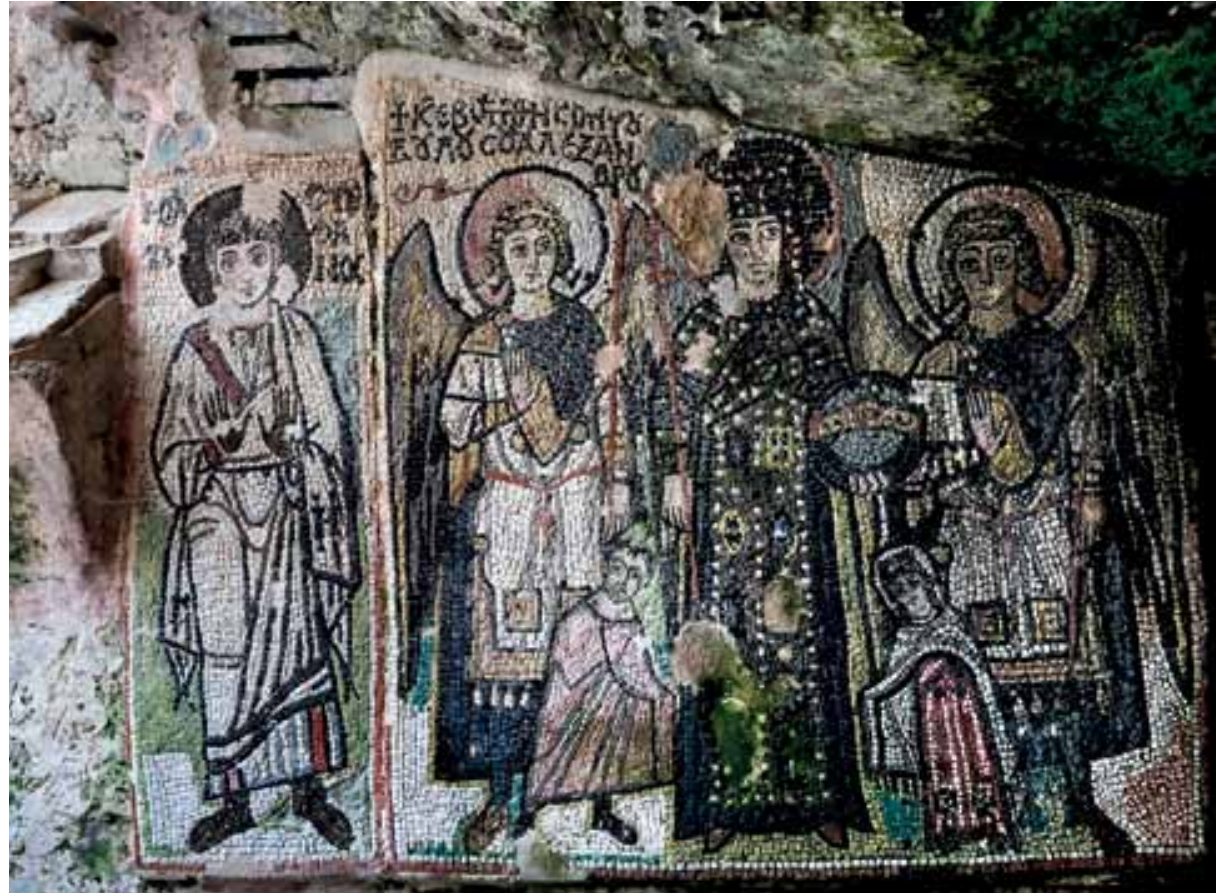

\section{Différents thèmes scientifiques explorés dans les volumes Corpus}

\section{Technique}

L'étude systématique du contexte architectural (sols, murs, mobilier) autour des mosaïques permet d'étayer les phasages observés dans les sols et d'aider à la compréhension de l'histoire de l'occupation de chaque édifice. Des relevés détaillés des sols aident à établir le diagnostic de leur état de conservation et des restaurations antiques. Ils autorisent des restitutions, bien souvent nécessaires pour une meilleure lecture des fragments préservés. Le travail conjoint du chercheur et du restaurateur donne lieu à de nombreuses vérifications techniques sur le terrain (sols antérieurs, restaurations antiques, superpositions, sondages, tracés préparatoires, composition des matériaux).

Nous avons pu constater, en étudiant les pavements in situ, que c'est bien souvent par l'étude des détails techniques, parallèlement à l'iconographie, que l'on peut discerner les mains et les ateliers de production (Raynaud 2008). L'observation de possibles empreintes, l'étude des mortiers et des supports, le relevé d'éventuels tracés préparatoires (visibles en cas de dépose) ou de limites de travail, l'analyse des matériaux composant les tesselles ${ }^{19}$ et de leur technique de pose sont riches de renseignements à la fois sur les ateliers, les conditions financières des commanditaires et le temps imparti à la réalisation des sols.

De même que les techniques, matériaux et recettes d'un même atelier évoluent peu, son répertoire iconographique est généralement assez répétitif, particulièrement pour les motifs secondaires pour lesquels sa liberté d'expression reste plus grande. C'est ainsi souvent par l'étude de la façon d'exécuter tel petit motif géométrique en apparence anodin ou répétitif, ou encore de « rendre » un oiseau que l'on repère des similitudes, des « tics de langage » communs entre deux pavements et donc des équipes ou des «mains » communes.

19 Locaux ou importés, remployés (marbres ou verres). Une prospection des carrières et roches locales est à l'occasion effectuée.

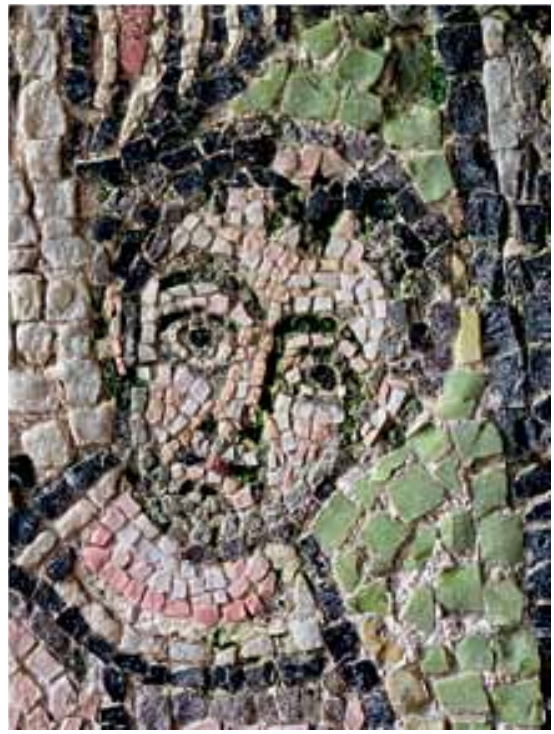

Figure 9

Durrës, mosaïque de la chapelle de l'amphithéâtre, ensemble et détail

(D. Dubois). 
Enfin, lorsqu'une équipe est suffisamment bien connue à travers une production importante, il est parfois possible de constater des anomalies, notamment dans le choix des sujets. Ainsi, l' «atelier d'Ohrid » maitrisait parfaitement son répertoire favori : les trames en quadrillage ou à médaillons dont chaque case renferme un animal, avec une forte prédilection pour les oiseaux ou les poissons. Les motifs de bordures généralement employés sont souvent les mêmes d'un site à l'autre (Muçaj - Raynaud 2005; Tutkovski 2014). Aussi lorsque l'on rencontre un motif ou une scène qui sort de l'habitude, et lorsque ce sujet est traité avec une certaine maladresse, on est tenté d'y reconnaître un thème imposé. C'est le cas des figures humaines réalisées à Byllis par l'« atelier d'Ohrid », dans l'exonarthex de la basilique B (Fig. 4D) et le sanctuaire de la basilique C. La question du commanditaire et de son influence sur les choix iconographiques se pose alors, même lorsque l'atelier de mosaïstes est réputé et de bonne maîtrise.

Des programmes archéométriques spécifiques sont menés parallèlement aux travaux sur le terrain :

- une série d'analyses de tesselles en verre ${ }^{20}$ est en cours (2014-2015) sur des prélèvements réalisés sur une dizaine de sites albanais. L'objectif de l'étude est de préciser un certain nombre de données, à travers des échantillons d'un nombre significatif de sites d'une même région et d'une même époque : l'évolution des techniques de fabrication du verre (pigments, opacifiants), la localisation des ateliers de verriers, le repérage du remploi de tesselles de verre ou de refontes, et des informations sur la datation, la circulation du verre et des mosaïstes, en particulier au $\mathrm{VI}^{\mathrm{e}}$ siècle où leur technique évolue. Quelques tesselles à feuilles d'or feront également l'objet d'une analyse toute particulière (Durrës, Lin, Elbasan);

- l'étude des mortiers et pierres est envisagée, également en collaboration avec Elisabetta Neri et des laboratoires d'analyses physico-chimiques ;

- l'étude physique des matériaux par lames minces et macro photographies, d'ores et déjà pratiquée, sera menée systématiquement (D. Dubois et E. Neri), visant à établir une véritable « matériothèque » des mosaïques d'Albanie.

\section{Ateliers}

C'est une chance très rare de pouvoir étudier les pavements d'une région entière. Grâce à un inventaire systématique, cela permet d'établir des comparaisons significatives et, à terme, d'identifier la production d'ateliers, locaux ou itinérants. Le fonctionnement, la circulation et les caractéristiques techniques de chacun commencent à apparaître plus clairement au fur et à mesure de l'avancée du projet. Les restaurateurs albanais, en particulier Agron Islami, travaillent sur ces pavements depuis plusieurs décennies. Leur connaissance approfondie de ces vestiges et des restaurations menées durant ces années complète le travail du Corpus.

L'étude déjà réalisée sur le terrain depuis 1999, à Byllis, Saranda, Lin, Elbasan, Tepe Elbasan et Butrint, commence donc à révéler les secteurs de travail de divers ateliers. Plusieurs d'entre eux ont été identifiés, équipes locales ou en provenance de grands centres urbains, qui ont travaillé ensemble à la confection

20 E. Neri est responsable du projet, en collaboration avec le laboratoire d'analyse du C2RMF (responsable I. Biron, au laboratoire du musée du Louvre) utilisant la méthode très précise du PIXEPIGE (rayons Gamma et X). L'étude et l'interprétation ont commencé en 2014 et se poursuivent en 2015. Elle participe aux travaux de terrain du Corpus d'Albanie depuis 2014. 
des mosaïques, comme dans les thermes et autres monuments publics romains de Butrint, ou encore dans les églises protobyzantines des sites étudiés.

La bonne connaissance de la «manière » et du savoir-faire du principal atelier de Byllis a permis de situer son origine dans la région d'Ohrid, en République de Macédoine ${ }^{21}$. À l'époque justinienne, l'« atelier d'Ohrid » ${ }^{22}$, dont la production a donc largement débordé les alentours du lac (Bitrakova-Grozdanova 2006; Tutkovski 2012; Tutkovski 2014) est intervenu dans les églises protobyzantines de Byllis, au Sud de l'Albanie, pour y effectuer la plupart des mosaïques (Muçaj - Raynaud 2005; Raynaud 2014). Il a fortement influencé les ateliers locaux d'importance secondaire qui y travaillaient simultanément ${ }^{23}$. L'étude prochaine de la mosaïque d'Arapaj, sans doute aussi du même atelier, devrait s'avérer une clef importante dans la connaissance de cet atelier itinérant. D'autres parallèles seront cherchés avec les mosaïques conservées à Heraclea Lyncestis et Stobi, qui comme Arapaj, Lin et Ohrid, se trouvent sur la via Egnatia. Un travail commun avec des chercheurs travaillant sur les mosaïques de l'actuelle République de Macédoine ${ }^{24}$ et de la République du Kosovo ${ }^{25}$ enrichira considérablement la connaissance de cet atelier prestigieux.

Enfin, un autre atelier de mosaïstes, originaire de Nicopolis (HellenkemperSalis 1987; Poulter 1995; Zachos 2007) ${ }^{26}$, capitale d'Epirus Vetus, a principalement œuvré sur le littoral ionien et adriatique, dans le nord de la Grèce, à Corfou (Kiourtzian 2013-2014) et dans le sud de l'Albanie, à Butrint et peut-être Saranda. L'aire d'influence de cet atelier, actif à l'époque justinienne, est encore peu connue. La recherche n'en est qu'à ses débuts.

On a pu constater que les ateliers « importés » des grands centres se déplaçaient pour effectuer la décoration d'un ensemble d'espaces ou celle d'un lieu particulièrement important, comme la mosaïque placée au-dessus de la tombe des martyrs d'Arapaj ou le grand baptistère de Butrint. Au contraire, les ateliers locaux étaient, semble-t-il, sollicités pour réaliser la décoration de petits espaces. Les premiers avaient une vision d'ensemble de la décoration à réaliser en une ou deux saisons. La planification par l'atelier d'Ohrid de l'ouvrage à réaliser se traduit dans les mosaïques par une mise en scène et une organisation synthétique du décor de chaque espace, souvent avec une bordure enveloppante et une recherche de symétrie (Fig. 4A): le choix et l'emplacement des sujets représentés semblent avoir un rôle à jouer dans le déroulement de la liturgie... Ce savoirfaire ne se rencontre pas de façon aussi aboutie dans le cas du travail d'ateliers «locaux », qui interviennent ponctuellement, et non pour élaborer un programme

21 Les mosaïques d'Ohrid ont été étudiées dès les années 1970 (Bitrakova-Grozdanova et al. 1975). La connaissance de la production des divers ateliers a été reprise et mise à jour tout récemment, dans l'excellent ouvrage déjà cité (Tutkovski 2014, en macédonien ; nous envisageons de faire traduire ce livre).

22 Il a réalisé les sols du tétraconque d'Ohrid, de Saint-Erasme, de Studencišta, de Radolišta et de Lin.

23 Une communication faisant le point sur les ateliers de mosaïstes en Epire et en Macédoine a été présentée par les auteurs au colloque international Illyrie Méridionale 6, qui s'est tenu à Tirana en mai 2015 (Raynaud - Islami, in print).

24 Miško Tutkovski, conservateur à Stobi, s'est joint à notre équipe du Corpus des mosaïques d'Albanie en 2014. Nous étudions l'éventualité de la création d'un Corpus des mosaïques de Macédoine, sur les mêmes normes que celle du Corpus d'Albanie, qui complèterait notre connaissance des mosaïques de cette région des Balkans encore souvent méconnue.

25 Des mosaïques de l'actuel Kosovo s'avèrent être sans doute des productions de l'atelier dit d'Ohrid, comme celles de la villa de Nerodimlje (Djurić 1994). Nous prévoyons des voyages d'études sur les différents sites et une prise de contact avec les archéologues kosovars.

26 L'atelier de mosaïstes de Nicopolis avait été reconnu par Ernst Kitzinger dès la fouille (Kitzinger 1951; revu dans Kitzinger 2002) ; identification reprise et argumentée entre autres par J.-P. Sodini (Sodini 1970) et D. Pallas (Pallas 1977). 

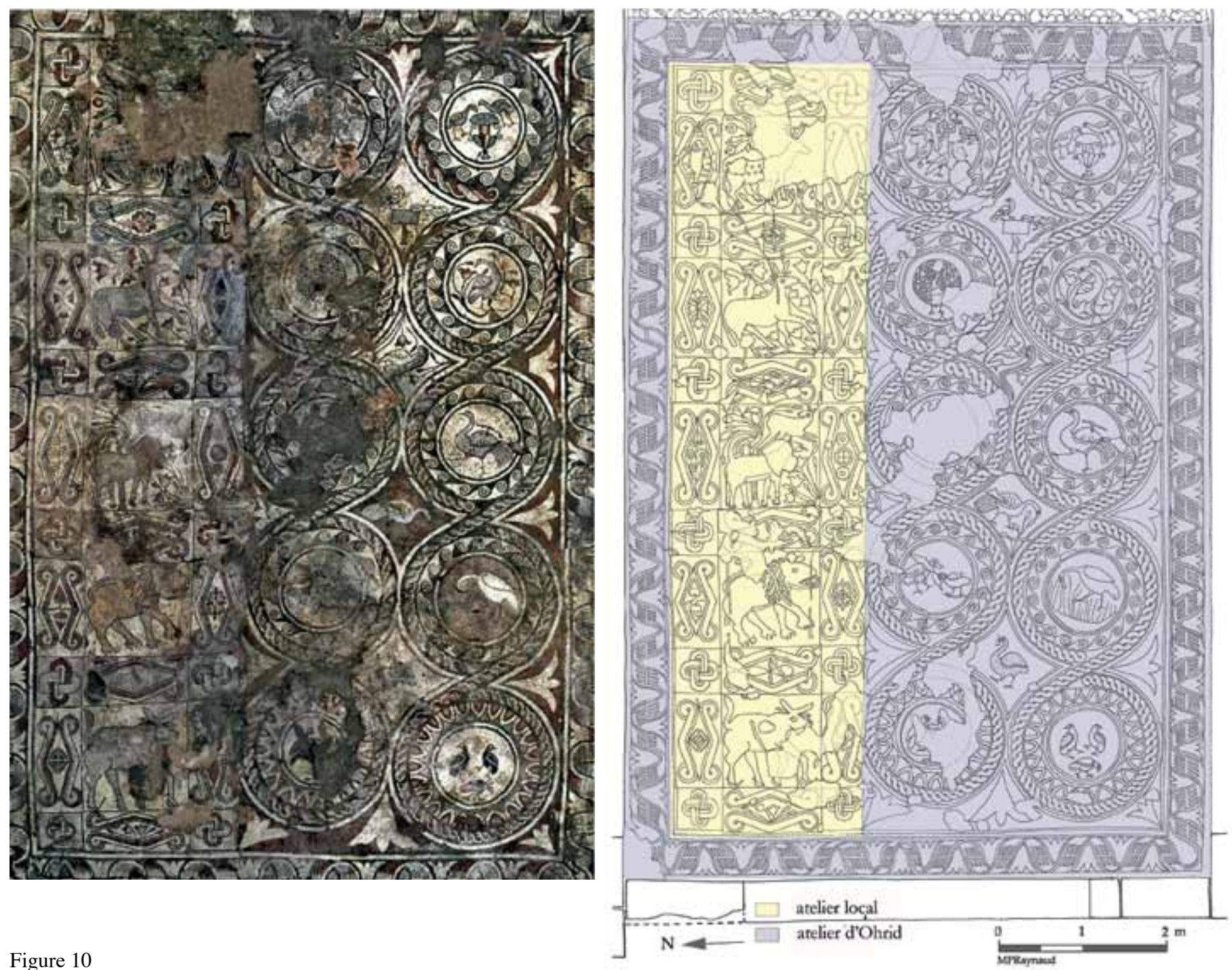

Figure 10

Byllis, nef centrale de la basilique A, œuvre simultanée de deux ateliers (M.-P. Raynaud).

iconographique à l'échelle d'un ou plusieurs bâtiments. Des exemples suggestifs illustrant ces observations ont été observés à Byllis et à Saranda.

On a plusieurs fois décelé le travail de plusieurs équipes d'artisans simultanément sur un même site : doit-on supposer un rôle formateur des équipes « importées » sur les ateliers « locaux »? On a parfois pu relever le travail de deux équipes différentes à l'intérieur d'un même espace, comme dans la nef centrale de la basilique A à Byllis (Fig. 10).

Particularité peu courante, on a constaté à plusieurs reprises le remploi de restes de mosaïques d'un bâtiment antérieur dans de nouveaux pavements de l'époque protobyzantine : même lacunaires, les fragments anciens ont été gardés visibles en place, englobés au même niveau dans un nouveau décor dont les lignes ne tiennent généralement aucun compte de ces restes antérieurs. Cette façon de respecter et de réutiliser des pavements anciens (éventuellement de les restaurer) sans les copier est inusitée. Cet esprit de conservation reflète-t-il un souci d'économie, une volonté de gagner du temps ou de ne pas retarder trop longuement le déroulement du culte? Ou témoigne-t-il de l'estime des artisans ou commanditaires pour un travail ancien apprécié (Daszewski 2003)? L'absence d'ateliers vraiment spécialisés à certaines périodes sur un site isolé pourrait aussi expliquer ces remplois qui limitaient la surface à décorer. La nef centrale de la 


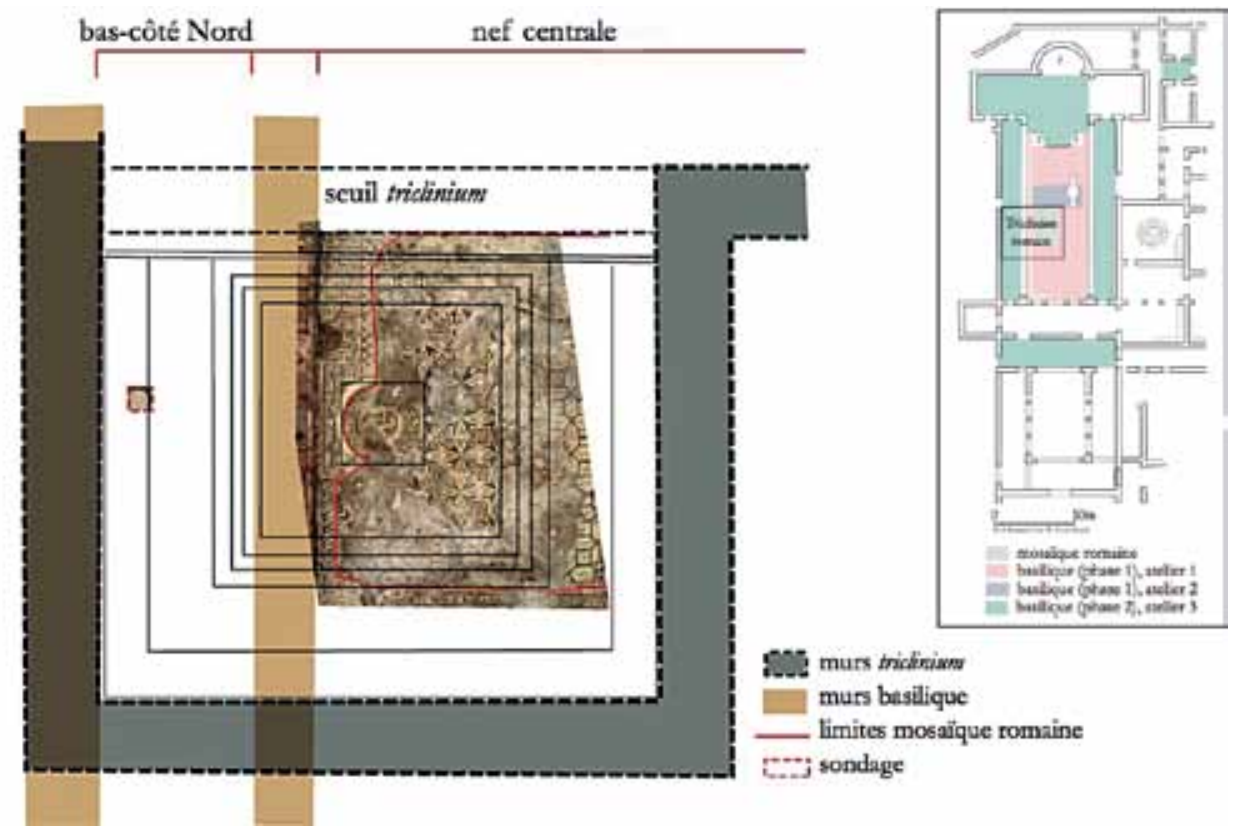

Figure 11

Byllis, nef centrale de la cathédrale (basilique B) avec remploi d'un fragment du triclinium romain antérieur (M.-P. Raynaud,

D. Dubois).

basilique $\mathrm{B}$ de Byllis, réalisée dans une première phase de décoration par des ateliers locaux, comporte un fragment de la mosaïque d'un triclinium romain antérieur (Islami - Raynaud 2014), curieusement préservé sur le côté de la nef (Fig. 11). Dans la phase suivante, l'« atelier d' Ohrid », chargé de décorer les nefs latérales, l'exonarthex, le transept et le sanctuaire, furent chargés d'unifier la nef centrale par l'insertion d'une large bordure unitaire ${ }^{27}$, sans toutefois parvenir à atténuer l'aspect hétéroclite des tapis juxtaposés du champ.

À Saranda, lors du passage de la synagogue rectangulaire au plan basilical avec changement d'axe, les sols d'origine ont continué de servir, et seul l'agrandissement de l'espace fut mosaïqué de neuf. On observe ensuite, sur une longue période, la pose progressive de petits panneaux, au gré, semble-t-il, des apports financiers de donateurs, pour restaurer le sol au fur et à mesure de sa dégradation (Raynaud 2012) ${ }^{28}$. Il résulte de cette façon de juxtaposer des panneaux, exécutés par des artisans différents, une impression de décor très disparate, sans unité, à « effet patchwork». Elle semble être le fait d'ateliers locaux successifs.

\section{Iconographie, pèlerinages}

L'étude d'ensemble des pavements à l'échelle d'une région nous donnera, en outre, l'occasion d'en développer les particularités iconographiques, liturgiques, techniques et de tirer des conclusions économiques de ces observations. Si, aux $\mathrm{V}^{\mathrm{e}}-\mathrm{VI}^{\mathrm{e}}$ siècles, le répertoire figuré et symbolique est fidèle à celui de l'ensemble de la Méditerranée, une étude iconographique précise pourrait dégager les particularités albanaises et les mettre en perspective dans le contexte plus large des Balkans. C'est dans ce sens qu'il a été pris contact avec un chercheur spécialiste ${ }^{29}$, qui étudiera le répertoire des mosaïques protobyzantines d'Albanie dans

27 En tronquant sans état d'âme le petit fragment romain jusque-là soigneusement préservé.

28 Dans la basilique-synagogue, les panneaux juxtaposés de la mosaïque ont été posés en sept phases successives, au-dessus des deux phases hellénistiques et romaines.

29 A.-O. Poilpré, iconographe, maître de conférence en Histoire de l'Art-Université Paris I PanthéonSorbonne-UFR 03, Institut d'Art et d'Archéologie, Laboratoire HiCSA, a commencé l'étude des mosaïques de Butrint. 
un cadre géographique et un contexte plus vastes et le confrontera aux autres expressions de l'art à cette époque.

Ayant constaté que plusieurs tombes privilégiées semblaient à l'origine de construction d'églises protobyzantine, parfois recouvertes de mosaïques, nous avons souhaité développer une recherche sur le lien entre les pavements et les tombes. Nous citerons des églises de Byllis (basilique D), Butrint (basilique de l'Acropole), Arapaj, Elbasan (Fig. 7A), Tepe Elbasan ou Lin (inscription audessus de la tombe). L'importance de ces sépultures faisait du lieu un but de pèlerinage, caractérisé par une architecture adaptée à la circulation de foules. Une étude comparative par un spécialiste permettra de mieux connaître les itinéraires des pèlerins dans la région, et les différentes solutions architecturales et funéraires trouvées pour répondre à cette vocation martyriale ${ }^{30}$. Une meilleure connaissance du contexte des pèlerinages de la région du lac d'Ohrid, autour du culte de saint Erasme d'Antioche, devrait également permettre de mieux cerner son importance.

\section{La série du Corpus des mosaïques d'Albanie}

Conçu comme la publication d'une série de livres scientifiques, le Corpus des mosaïques d'Albanie fera connaître l'ensemble des pavements du pays aux spécialistes internationaux de la mosaïque. Outre le catalogue systématique qui forme le corps du livre, selon une fiche descriptive proche de celle établie pour le Corpus de Turquie (Annexe), celui-ci présentera une brève description de chaque monument et de son histoire, l'historiographie des recherches faites sur chaque pavement, ainsi qu'un compte-rendu des restaurations antiques et modernes. Une synthèse conclura chaque volume, faisant le bilan du phasage chronologique des pavements, des ateliers de mosaïstes repérés, des comparaisons et influences, de l'étude stylistique et iconographique et des particularités techniques observées. Les inscriptions sur mosaïque feront l'objet d'une étude épigraphique particulière. Des analyses archéométriques complèteront éventuellement l'ensemble.

Le Corpus des mosaïques d'Albanie respectera les préceptes des autres publications de ce type ${ }^{31}$, et en particulier les dernières parutions en date, du Corpus of the Mosaics of Turkey. Les volumes offriront les mêmes caractéristiques et adopteront une mise en page similaire, souple et riche, avec de nombreuses illustrations en couleur ${ }^{32}$. Seront présentés systématiquement, en parallèle au catalogue, des plans des sites et édifices, des relevés détaillés des mosaïques et des restitutions, des schémas de phases, des montages photographiques, des clichés de détail et des documents d'archives. Nous pensons publier le premier volume en 2016-17; les suivants devraient se succéder rapidement et régulièrement.

Les participants prévoient en outre la publication de fréquents articles (communications individuelles ou collectives à des colloques, tables rondes, revues nationales et internationales) qui présenteront régulièrement les résultats des recherches ; des rapports annuels seront envoyés aux diverses institutions participantes.

30 Yuri Marano a été contacté pour prendre en charge cette partie de l'étude, en lien avec l'étude des pavements.

${ }^{31}$ Les publications suivront les normes mises en place par l'AIEMA, association internationale pour l'étude de la mosaïque antique.

32 Le Labex Resmed et la Butrint Foundation participeront à l'édition du premier volume. Nous espérons qu'ils prolongeront leur soutien financier pour la fabrication des différents volumes. 


\section{Aide au développement touristique}

L'Albanie s'ouvre depuis quelques années à un tourisme toujours croissant. Une des ambitions du programme du Corpus est de mieux faire connaître ce patrimoine à un public de plus en plus demandeur, tout en respectant la sécurité et les principes internationaux de conservation et de présentation des sites. Au niveau local, la documentation systématique réunie sur les mosaïques d'une région entière, toutes époques confondues, offrira un soutien scientifique à l'aménagement des sites ou des musées (confection de panneaux signalétiques clairs et didactiques), à l'élaboration de plaquettes destinées au grand public et à la préparation d'expositions temporaires. Nous espérons que la publication du Corpus des mosaïques d'Albanie stimulera les efforts d'aménagement des sites pour un public de plus en plus nombreux.

À l'échelle d'un pays de dimensions relativement modeste comme l'Albanie, nous pouvons envisager la réalisation du Corpus des mosaïques d'Albanie comme le travail de toute une équipe, et non la juxtaposition d'entreprises individuelles. Le nom de chaque participant sera associé aux publications, site après site. Si l'entreprise de documentation systématique in situ est un préalable à toute vision d'ensemble d'une région et à toute recherche ciblée, les recherches en bibliothèque et en archives permettent de compléter l'étude historiographique de chaque monument et de prendre en compte les pavements perdus ou inaccessibles. À l'issue de l'inventaire méthodique des mosaïques, de l'étude de leur répertoire, des matériaux utilisés, du style, de la technique, notre perception des diverses équipes de mosaïstes deviendra plus pertinente. Des études similaires dans les pays environnants (Kosovo, Macédoine, Bulgarie, Croatie, Nord de la Grèce) permettraient d'étayer utilement ce travail de fond et de compléter notre connaissance de la rive Est de l'Adriatique et de la circulation des courants culturels dans le secteur des Balkans.

\section{Bibliography - Kaynaklar}

Anamali 1974

Skënder Anamali, “Mozaikët e bazilikës paleokristiane të Linit (Pogradec)”, Iliria 3: 329-342.

Bitrakova-Grozdanova et al. 1975

Vera Bitrakova-Grozdanova - Dordi Krestevski - Petar Alcev, Старохристијански споменици во Охридско (Les monuments paléochrétiens de la région d'Ohrid), Ohrid (Institut pour la protection des Monuments de la culture, musée national d'Ohrid).

Bitrakova-Grozdanova 2006 Vera Bitrakova-Grozdanova, "Lychnidos entre l’Orient et l'Occident paléochrétiens”, Actes du XIV CIAC, Vienne 1999: 213-220.

Bowden 2007

William Bowden, "Diaporit - una villa romana e un monastero tardo", Groma 1: 101-106.

Bowden - Hodges 2011

William Bowden - Richard Hodges (eds.), Butrint 3, Excavations at the Triconch Palace. Butrint Archaeological Monographs 3, Oxford, Oakville (Conn.), Oxbow Books for the Butrint Foundation.

Bowden - Përzhita 2014 William Bowden - Luan Përzhita, "The Roman villa and Early Christian complex at Diaporit", International Congress of Albanian archaeological Studies; Proceedings of 65th anniversary of Albanian Archaeology (2122 nov., Tirana 2013), Tirana, 469-482.

Budina - Korkuti 1971 Dhimosten Budina - Muzafer Korkuti, "Mozaikë të rinj të zbuluar në Onkesmin e lashtë (Sarande)", Studime Historike 1: 207-247.

Bushi - Xhaferaj - Behari 2014 Skënder Bushi - Era Xhaferaj - Altin Behari, "Mozaiku paleokristian në Kalanë e Beratit. Raport Paraprak", Candavia 4: 205-214.

Cerova - Hobdari - Islami 2011 Ylli Cerova - Elio Hobdari - Agron Islami, "Bazilika paleokristiane extra-muros n. Scampis (Elbasan); Rezultatet e gërmimeve 2007-2009,(La basilique paléochrétienne extra-muros de Scampis (Elbasan); Fouilles 2007-2009)", Candavia 3: 105-130.

Chevalier et al. 2003

Pascale Chevalier - Marie-Patricia Raynaud - Catherine Vanderheyde - Manuela Wurch-Zozelj - Nicolas Beaudry - Skënder Muçaj - Jean-Pierre Sodini, "Trois basiliques et un groupe épiscopal des $\mathrm{v}^{\mathrm{e}}$ - $\mathrm{vI}^{\mathrm{e}} \mathrm{s}$. réétudiés à Byllis (Albanie)”, Hortus Artium Medievalium 9: 155-165. 
Daszewski 2003

Décor I 1985

Décor II 2002

de Maria - Zaccaria 2005

de Maria - Podini 2009

Dimo - Lenhardt - Quantin 2007 Vangjel Dimo - Philippe Lenhardt - François Quantin (eds.), Apollonia d'Illyrie 1. Atlas archéologique et historique, coll EfR 391, Rome.

Djurić 1994

Foerster et al. 2004

Greenslade 2013

Greenslade - Çondi 2010

Guimier-Sorbet 1993

Hansen - Hodges - Leppard 2013 Inge Lyse Hansen - Richard Hodges - Sarah Leppard (eds.), Butrint 4: The Archaeology and Histories of an Ionian Town. Butrint Archaeological Monographs 3, Oxford Oakville (Conn.), Oxbow Books for the Butrint Foundation.

Hellenkemper-Salis 1987

Hidri - Hidri 2011

Hobdari -- Cerova 2013

Hodges - Bowden - Lako 2004

Hoti - Shehi - Santoro 2012

Islami - Raynaud 2014

Kiourtzian 2013-2014

Kitzinger 1951

Kitzinger 2002

Komata 1984

Kosta - Çikopano 1990

Manière-Lévêque - Parrish - Raynaud 2012

Anne-Marie Manière-Lévêque - coll. David Parrish - Marie-Patricia Raynaud, Corpus of the Mosaics of

Anne-Marie Manière-Lévêque - coll. David Parrish
Turkey. Vol. II-Lycia, Part 2-The West Area, Istanbul.

Wiktor A. Daszewski, "Remarques sur l'attitude des anciens vis-à-vis de la restauration des mosaïques", ICCM Conference (Arles-Saint-de- Romain en Gal 1999), Arles: 245-254.

Catherine Balmelle - Michèle Blanchard-Lemée - Janine Christophe - Jean-Pierre Darmon - Anne-Marie Genier-Sorbets - Henri Lavagne - Richard Prudhomme - Henri Stern, Décor I, Le décor géométrique de a mosaique romaine, 1. Compositions isotropes et linéaires, Paris, Picard.

Raynaud, Décor II, Le décor géométrique de la mosaïque romaine, compositions centrées, Paris, Picard.

Sandro de Maria - Mirco Zaccaria, "Le nuove ricerche, in saggi di scavo nell'area della basilica bizantina", Gjongecaj (eds.): 89-96.

Sandro de Maria - Marco Podini, "La basilica paleocristiana di Phoinike (Epiro): dagli scavi di Luigi M. Ugolinialle nuove ricerche",Attidel Convegnointernazionale,Phoinike,Bologna 2007,R.Farioli-CampanatiC. Rizzardi - P. Porta - A. Augenti - I. Baldini Lippolis (eds.): 207-228.

Srdjan Djurić, "Mosaic of Philosophers in an Early Byzantine Villa at Nerodimlje", in La mosaïque Grécoromaine VI (VI Colloquio internacional sobre mosaico antoguo, Palencia, Merida 1990): 123-134.

Gideon Foerster - Ehud Netzer - Kosta Lako - Etleva Nallbani, "Sinagoga e Sarandës (Anchiasmos) në Antikitetin e vonë", Candavia 1: 32-48.

Simon Greenslade, "The Vrina plain settlement between the $1^{\text {st }}-13^{\text {th }}$ century", in Butrint 4 . The Archaeology and Histories of an Ionian Town: 123-163.

Simon Greenslade - Dhimitër Çondi, "Recent Excavations on the Vrina Plain, Butrint: A preliminary report on the archaeological sequence from the $1^{\text {st }}$ to $6^{\text {th }}$ century", Actes du $\mathrm{V}^{\mathrm{e}}$ colloque Illyrie Méridionale, P. Cabanes - J.-L. Lamboley (eds.), Paris, de Boccard: 265-277.

Anne-Marie Guimier-Sorbet, "La mosaïque hellénistique de Dyrrachium et sa place dans la série des mosaïques grecques à décor végétal”, Actes du II colloque Illyrie Méridionale, P. Cabanes (ed.), Paris, de Boccard: 135-141.

Gisela Hellenkemper-Salis, "Zu stil und Ikonographie in den frühbyzantinischen Mozaiken von Nikopolis", Proceedings of the $1^{\text {st }}$ International Symposium on Nikopolis, 1984, E. Chrysos (ed.), Preveza: Municipality of Preveza: 295-309.

Hava Hidri - Sali Hidri, Die frühchristliche basilika in Arapaj/Durrës (Albanien), band 20, Vienne.

Elio Hobdari - Ylli Cerova, “Bazilika paleokristiane extra-muros në Scampis (Elbasan)”, Iliria 36: 451-456.

Richard Hodges - William Bowden - Kosta Lako (eds.), Byzantine Butrint: Excavations and Surveys 19941999, Oxford: Oxbow Books for the Butrint Foundation.

Afrim Hoti - Eduard Shehi - Sara Santoro, "Nuovi mosaici romani da Dyrrachium (Albania)- scoperte del 2003-2005”, La mosaïque Gréco-romaine X (Conimbriga 2005), Lisbonne: 593-608.

Agron Islami - Marie-Patricia Raynaud, "Mozaikët e katedrales së Bylisit: vëzhgime të reja në terren dhe të dhëna kronologjike", Monumentet 52-1: 145-50.

Georges Kiourtzian, "Les inscriptions de la basilique de Iovianos à Corfou", Cahiers archéologiques 55: 5-16.

Ernst Kitzinger, "Studies on Late Antique and Early Byzantine Floor Mosaics. I. Mosaics at Nikopolis", Dumbarton Oaks Papers 6: 82-122.

Ernst Kitzinger, "Studies on Late Antique and Early Byzantine Floor Mosaics. I. Mosaics at Nikopolis", in Studies in Late Antique Byzantine and Medieval Western Art, I, London: 189-198.

Damian Komata, "Bazilika paleokristiane e Mesaplikut”, Iliria 14/1: 183-197.

Sotir Kosta - Uilliam Çikopano, "Mozaiku i bazilikës paleokristiane në Tepe të Elbasanit. Vlerat dhe restaurimi", Monumentet 39/1: 129-138. 
Marika - Nesturi 2014 Zamir Marika - Enkelejda Nesturi, “Arti muziv dhe mozaiku i Asklepit - Butrint. Personifikimi dhe miti gjarprit (asklepios) në Iliri dhe Epir”, Monumentet 52-1: 135-44.

Meksi - Ceka $1971 \quad$ Aleksandër Meksi - Neritan Ceka, "Bazilika paleokristiane në Tepe (Elbasan)”, Buletin Arkeologjik: 184-190.

Mitchell 2006

John Mitchell, "Strategies for salvation: the mosaic pavement of the Triconch Church at Antigoneia", New Directions in Albanian Archaeology. Studies presented to Muzafer Korkuti, L. Bejko - R. Hodges (eds.), ICAA, Tirana: 261-276.

Mitchell 2008 John Mitchell, Pagëzimorja e Butrintit dhe Mozaikët e saj, The Butrint Baptistery and its Mosaics, London-Tirana, Butrint Foundation.

Morlier - Raynaud 2007 Hélène Morlier - Marie-Patricia Raynaud, "A survey of the Previous Corpusses and Techniques of Illustration in order to Prepare the Publication of the Corpus of Mosaics of Turkey”, III. Uluslararası Türkiye Mozaik Korpus sempozyumu bildirileri (Bursa 2006), M. Şahin (ed.), Bursa: 149-156, pl. 215-222.

Muçaj et al. 2004 Skënder Muçaj - Kosta Lako - Elio Hobdari - Yannis Vitaliotis, "Rezultatet e gërmimeve në Bazilikën e Shën Janit, Delvinë (2001-2003) [Résultats des fouilles de la basilique de Saint-Jean à Delvina (2001-2003)]”, Candavia 1: 93-124.

Muçaj-Raynaud 2005 Skënder Muçaj - Marie-Patricia Raynaud, "Les mosaïques proto-byzantines des églises de Byllis, un atelier", La mosaïque Gréco-romaine IX (Rome 2001), H. Morlier (ed.), Paris-Rome, 1: 383-398.

Muçaj - Hobdari - Vitaliotis 2005

Skënder Muçaj - Elio Hobdari - Yannis Vitaliotis, "Kisha mesjetare e Peshkëpisë (Nivicë), Sarandë [l'église médiévale de Pëshkëpi (Nivica), Saranda]", Candavia 2: 273-310.

Nallbani 2003

Etleva Nallbani, "Une nouvelle synagogue de l'Antiquité (tardive?) identifiée à Saranda”, Artium Medievalum 9: $167-172$.

Nallbani et al. 2011

Etleva Nallbani - Marie-Patricia Raynaud - Ehud Netzer - Gideon Foerster - Kosta Lako, "La synagogue de Saranda", Actes du colloque Inrap-Musée d'art et d'histoire du Judaïsme (Paris 2010), P. Salmona - L. Sigal (eds.), Paris: 63-73.

Omari 2006

Elda Omari, "Simbolët e krishtërimit në Mozaikun e bazilikës së Arapajt”, Hylli i Dritës 4: 46-51.

Omari 2012

Elda Omari, "The Mosaics with animals themes in Southern Adriatic between $4^{\text {th }}$ and $6^{\text {th }}$ century A.D.: decorative and iconographic schemes in comparison", JMR 5: 115-129.

Pallas 1977

Demetrios Pallas, Les monuments paléochrétiens de la Grèce découverts de 1959 à 1973, Rome.

Përzhita - Bowden 2011 Luan Përzhita - William Bowden, "Excavations at the Roman Villa and Early Christian Complex at Diaporit", Candavia 3: 5-40.

Podini - Meta - Silani 2007

Marco Podini - Albana Meta - Michele Silani, “La basilica paleocristiana”, Phoinike IV. Rapporto preliminare sulle campagne di scavi e ricerche 2004-2006, S. De Maria - Shpr. Gjongecaj (eds.): 31-58.

Poulter 1995

Andrew G. Poulter (ed.), Nicopolis ad Istrum: A Roman, Late Roman, and Early Byzantine City, JRA Monograph 8.

Raynaud 2008

Marie-Patricia Raynaud, “Quelques remarques à propos de l'étude des sols in situ”, JMR 1-2: 83-101.

Raynaud 2009

Marie-Patricia Raynaud, Corpus of the Mosaics of Turkey, Xanthos, the East Basilica. Uludağ University Mosaic Research Center Series 1. Bursa, Uludağ University Press.

Raynaud 2012

Marie-Patricia Raynaud (collaboration Gideon Foerster - Kosta Lako - Etleva Nallbani - Ehud Netzer), "Une synagogue transformée en église à Saranda (Albanie)", La Mosaïque Gréco-romaine X (Conimbriga 2005): 687-701, pl. XXVI.

Raynaud 2014

Marie-Patricia Raynaud, "Corpus des mosaïques d'Albanie”, Proceedings of the international Congress of albanian archaeological Studies- 65th Anniversary of Albanian Archaeology (21-22 Nov., Tirana 2013), L. Përzhita - I. Gjipali-G. Hoxha - B. Muka (eds.), Tirana: 547-560.

Raynaud - Islami in print Marie-Patricia Raynaud - Agron Islami, “Ateliers de mosaïstes en Albanie. Sédentaires ou itinérants ?”, Colloque Illyrie Méridionale VI (Tirana, mai 2015).

Skenderaj $2014 \quad$ Altin Skenderaj, "Nouvelles données sur les sols mosaïqués d'Apollonia d'Illyrie”, Proceedings of the international Congress of albanian archaeological Studies- 65 $5^{\text {th }}$ Anniversary of Albanian Archaeology (21-22 Nov., Tirana 2013), L. Përzhita - I. Gjipali - G. Hoxha - B. Muka (eds.), Tirana: 353-366.

Sodini 1970 Jean-Pierre Sodini, "Mosaïques paléochrétiennes de Grèce", Bulletin de Correspondance hellénique 94: 699-753.

Tutkovski 2012

Miško Tutkovski, "Newly discovered Mosaics in the Tetraconchal Church at Plaošnik” Patrimonium: 139-148.

Tutkovski 2014

Miško Tutkovski, Ранохристијанските мозаици од Охрид, Skopje.

Zachos 2007

Konstantinos L. Zachos (ed.), Nicopolis B, Proceedings of the Second International Nicopolis Symposium (2002). 
Annexe

\section{FICHE DESCRIPTIVE POUR LE CORPUS DES MOSAÏQUES D'ALBANIE ${ }^{33}$}

Cette fiche est utilisée pour les mosaïques conservées ou perdues.

Lorsque l'on parle d'un édifice contenant plusieurs mosaïques, décrire d'abord l'édifice en général (sa fonction) et donner des détails pièces par pièces avant de documenter le pavement. Donner un plan de l'édifice, avec numérotation des pièces. L'ordre de description des pièces suivra la circulation logique dans l'édifice.

$\mathbf{N}^{\circ}$ de Corpus: sur un même site, les mosaïques sont numérotées en continu, la numérotation se poursuivant au besoin d'un volume à l'autre.

Technique des revêtements: mosaïque de galets, opus tesselatum, opus vermiculatum, opus sectile, mosaïque pariétale, opus signinum...

Titre de la mosaïque: mentionné s'il existe.

Provenance: nom du site d'où provient le pavement.

Lieu de conservation: in situ, dans un musée, dans une collection publique ou privée, perdu ou connu par des archives.

Contexte architectural, situation, fonction et numéro de la pièce ; donner les numéros des autres mosaïques décorant l'espace. Décrire l'espace et ses relations avec les pièces voisines, donner la forme du pavement et sa situation (par exemple un seuil rectangulaire ou un panneau carré devant une abside). Signaler, s'ils existent, les autres décors architecturaux de la pièce (stuc, mosaïque pariétale ou de voûte, peinture, placage).

Dimensions: actuelles, et restituées si la mosáique est fragmentaires.

Matériaux: nature des matériaux employés, galets de rivière, calcaire ou autre type de pierre, terre cuite, marbre, verre. Description du support si possible.

Analyses: les résultats d'analyses archéométriques éventuelles seront mentionnés.

Couleurs: des matériaux, si possible selon la table de Munsell.

Densité (pour opus tesselatum): nombre moyen de tesselles au $\mathrm{dm}^{2}$, pris en plusieurs parties de la mosaïque, raccords, bordures, champ ou figures/motifs. Pour les opus vermiculatum on donnera une estimation au $\mathrm{cm}^{2}$.

Etat de conservation: complet, fragmentaire, traces de calcination...

- réparations antiques; restaurations modernes (description et rapide historiographie), urgence éventuelle d'une restauration.

- état actuel: toiture, mosaïque déposée, remise sur un nouveau support, consolidations, protection du pavement (ré-enfoui ou couche de sable ou autre), ou pavement détruit.

- en musée: numéro d'inventaire, nombre de panneaux, visible ou non, en dépôt.

33 Fiche établie par H. Morlier, D. Parrish et M.-P. Raynaud, pour le Corpus of the Mosaics of Turkey, à partir des modèles existant pour les différents corpus. 
Documentation d'archives: à donner si le pavement est perdu ou partiellement détruit. Lieu de conservation et type d'archives (document archéologique, note de terrain, photographie, aquarelle, croquis, plan, rapport manuscrit), précisant l'auteur, la date et le titre.

Description de la mosaïque: de l'extérieur vers l'intérieur (raccords, bordures, champ ou tapis, avec ou sans panneaux, seuil, panneaux de rallonge).

Pour les décors géométriques et végétaux, utiliser la terminologie et le renvoi aux planches des deux volumes en cinq langues du Décor I - II.

S'il y a plusieurs panneaux, mettre un croquis les numérotant.

Commentaire: Observations sur le dessin, la technique, l'iconographie, le style et les comparaisons.

Date: datation, par contexte archéologique, épigraphique ou numismatique. Datation par critères stylistiques.

Bibliographie: une bibliographie générale peut être donnée pour la partie concernant l'édifice ; pour chaque pavement, bibliographie complète avec toutes les références publiées concernant le pavement (utilisation des abréviations de l'AIEMA, que l'on trouve dans tout Bulletin de l'Association internationale pour l'étude de la mosaïque antique). Exemple : J. Russel, « Mosaics of Anemurium », AntK 23, 1976, 242-256 ; D. Levi, Antioch Mosaic Pavements, Princeton, 1947, 23, pl. 56.

Illustration : dans un même volume, les plans et dessins seront standardisés, échelle et légendes.

- Plan de l'édifice, dessin de la mosaïque dans ses murs, avec restitution si nécessaire, schémas divers.

- Photographies d'ensemble et photographies de détail (motifs, figures, restaurations). Documents d'archives. 